\title{
Doherty Power Amplifiers in Software Radio systems
}

\author{
F.M. Ghannouchi ${ }^{1}$, and K. Rawat ${ }^{2}$ \\ iRadio Laboratory, Department of Electrical and Computer Engineering, Schulich School of Engineering, University of \\ Calgary, Calgary, AB, Canada T2N1N4, fadhel.ghannouchi@ucalgary.ca ${ }^{1}$, krawat@ucalgary.ca²
}

\begin{abstract}
Power amplification in software defined radio needs reconfigurabilty as well as optimum performance in terms of linearity and efficiency to handle different modulation standards (and hence carrier and modulation bandwidth). Since digital predistortion is now widely accepted as highly suitable solutions for linearization in a reconfigurable perspective. Hence, this technique in conjunction with multi-band Doherty power amplifiers finds a potential solution for reconfigurable-multi-band software defined radio transmitter. This paper demonstrates the current state-of art for such transmitters, including a brief discussion about the available design methodology for the dual-band Doherty power amplifier.
\end{abstract}

\section{Introduction}

In the perspective of software-defined radio (SDR) architectures, the demand of multi-band transmitters is becoming important in order to provide reconfigurability and multi-operability. Moreover, for high data rate handling capability with low power consumption, the transmitter architecture should also be selected in order to obtain highefficiency and linearity to maintain the quality of service [1]. Since linearity and efficiency are the two main benchmarks for any transmitter topology [1], an optimal solution is being sought. Among these solutions, the dual-band predistortion based transmitter is effective, in terms of high linearity and digital baseband implementation, which provides reconfigurability [1]. For such transmitters, the power amplifier (PA) is operated at a certain average power backoff, which depends on the peak-to-average power ratio (PAPR) of the predistorted signal. To enhance the efficiency in this back-off region, Doherty PA is studied extensively and adopted in base station [3-4]. Based on these motivations, a multi-band transmitter with multi-band DPA and a digital predistortion can be sought as optimal solutions in terms of high efficient and linear architecture that can be implemented easily in the current RF/digital platform for the base-stations [5-6].

In this paper existing methodologies for the development of dual-band Doherty PA have been discussed including the implementation issues. Some results are also presented for the dual-band Doherty PA design and its linearization while operating at $1.96 \mathrm{GHz}$ and $2.425 \mathrm{GHz}$ bands using WCDMA and WiMax signals respectively.

\section{Multi-Band Transmitter Architecture \& Design Consideration}

The multi-band transmitters as proposed herein, need multi-band Doherty PA and reconfigurable digital predistortion techniques.

\section{A. Dual-band Doherty PA design}

The main efforts in Doherty PA design is the development of Doherty combiner and dual-band carrier and peaking amplifiers. Such structure utilizes dual-band transformers or impedance inverters analogous to their single-band architecture [3-4]. Three possible dual-band transformers can be used in passive circuits associated with the Doherty PA. Among these, Pi-type and T-type stub loaded transmission lines represents $90^{\circ}$ type transformers [7-8] as the electric length at both bands are $90^{\circ}$ or its odd multiple. However, Monzon transformer is a non- $90^{\circ}$ type, since the electric length at the two bands depends on the frequency ratio $\left(f_{2} / f_{1}\right)$ of the dual-band operation, where, $f_{1}$ and $f_{2}$ represents the first and second frequency of operation respectively [9]. For the T-type or Pi-type stub loaded transmission lines, the image impedance and overall electric length are the function of the characteristic impedance and electric length of the respective loaded transmission line and the stub loading it [7-8]. These image impedances and overall electric length of the Pi-type or T-type structures can be derived using their corresponding transmission line matrices (ABCD matrix) which can be found in [7], [8] respectively. Thus, the T-type or Pi-type structure to a certain extent of frequency-band represents a $90^{\circ}$ transmission line, whereas on the other hand Monzon transformer act as impedance inverter in a certain band of frequency. For a dual-band Doherty PA associated circuitry ,the use of these type of structures are attempted in some designs [10-11]. 


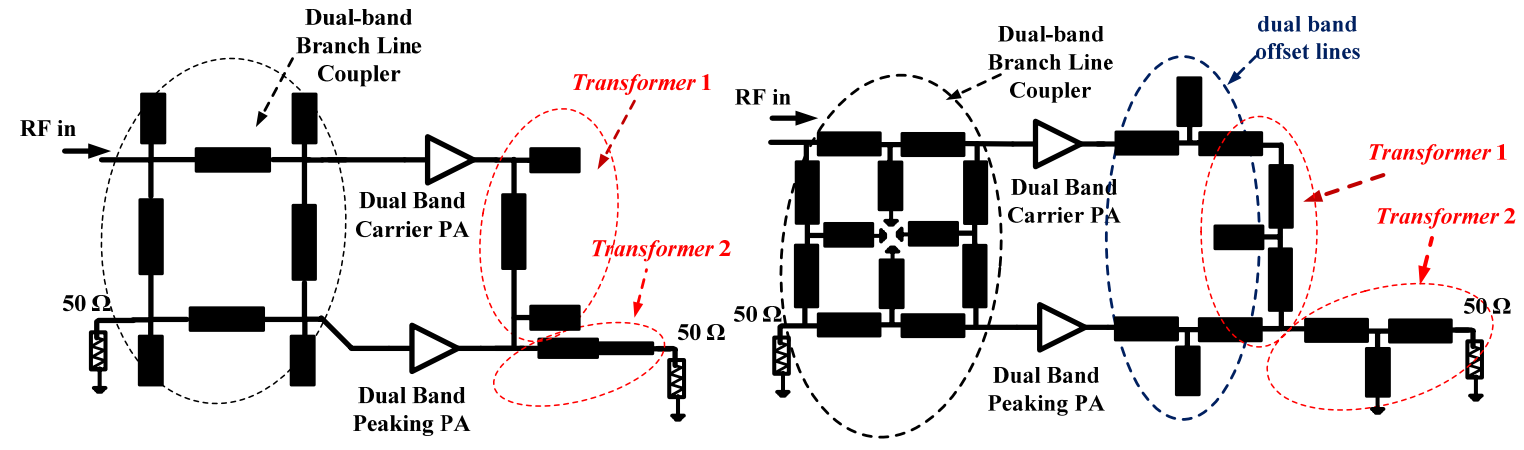

(a)

(b)

Fig. 2. (a) Dual-band Doherty architecture reported in [10], (b) dual-band Doherty configuration reported in [11].

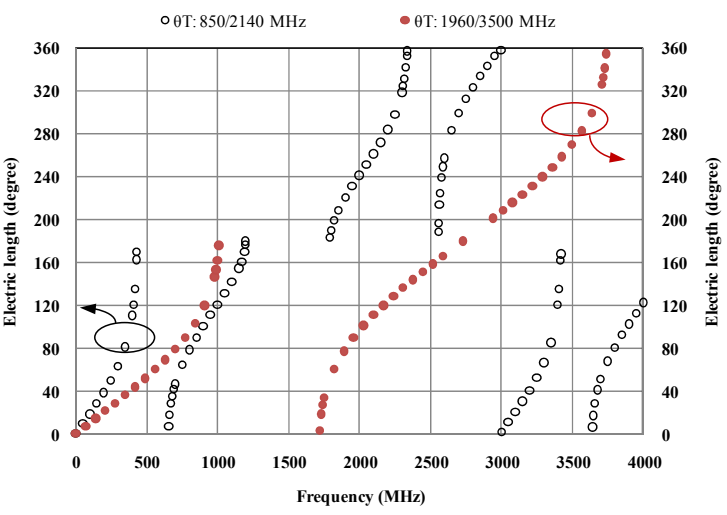

(a)

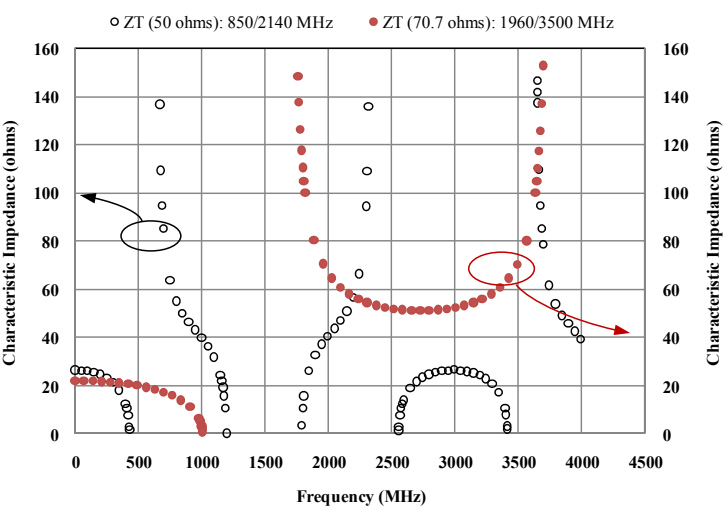

(b)

Fig. 3. Behavior of the T-type transformer with frequency: (a) effective electrical length, (b) effective characteristic impedance [8].

Fig. 2 represents the general architecture of these reported designs. The Doherty load modulation circuit proposed in [10] and as shown in Fig. 2 (a), utilizes Pi-type transformer and Monzon transformer, whereas for the input splitter a Pitype based branch-line hybrid has been used. The design methodology for Pi-type transformer and Monzon transformer can be obtained from [7], [9] respectively. Although, the design in Fig. 2 (a) does not use any dual-band output delay offset line to avoid leakage from carrier to peaking path in low power operation, this still provides an architectural solution for a Doherty PA. This delay line is very important for reducing leakage loss in the combiner and the performance improvement in its presence has already been established in the case of single-band operation [1], [4]. In fact, in absence of these delay offset lines, the designers in [10] are unable to test their Doherty PA in the concurrent mode due to this leakage of carrier current to the peaking current. In view of this fact, the architecture reported in [11] and as shown in Fig. 2 (b), has much advantage, where a dual-band T-type structure is used for dual-band delay line operation. However, the matching is also provided using T-type structure, where in the case of [10] (Fig. 2(a)), a proper dual-band matching design methodologies can be obtained from author's referred works. The T-type structure can be used for matching by shaping its frequency varying characteristic impedance and electric length. Such behavior is analyzed in details and reported in [8] for different frequency ratios. Fig. 3 shows this behavior of the T-type structure.

\section{B. Digital Predistortion based Linearization}

Several solutions have been proposed for the linearization techniques such as: feedback, feed forward, RF predistortion and baseband digital predistortion [1]. The selection of the appropriate technique, in most cases, is dependent on the application/standard, which dictates the targeted level of linearity to be reached. Despite the fact that most of these technologies can lead to acceptable levels of correction for the nonlinearity of PAs, the only compatible solution with SDR based transmitters is baseband digital predistortion, which can be considered as an enabling technology for third generation (3G) and beyond radio systems [2], [5].

Digital predistortion utilizes a behavioral model which can be seen as a black-box model that relates the input waveform to the output waveform, using the characterized amplitude AM/AM and AM/PM response of the PA [2]. 
In order to obtain the optimal back-off for the predistorter that would not change the peak and average power at which the PA characterization was performed, a power tracking scheme was implemented according to [12]. This ensured that the PA characteristics and the average power did not change significantly when the predistorter was introduced and that the peak-to-average power ratios (PAPRs) of the input and transmitted signals at the output of the linearized PA were approximately equal. A very small variation in average power could be tolerated, assuming the PA behavior herein was insensitive to power variation of less than $0.5 \mathrm{~dB}$ [12].

\section{Implementation and Performance}

An in-house dual band Doherty PA fabricated was tested for WCDMA and WiMAX signals at two different carrier frequencies. The performances of this Doherty PA in terms of drain efficiency and gain are shown in Fig. 4 and the results obtained are compared to the balanced amplifier ones. The Doherty PA is linearized individually at two bands in presence of two different signals, WCDMA and WiMax and the performance in terms of adjacent channel power ratio are shown in Fig. 5. These results show that the developed Doherty PA can work at both the frequencies and since the linearization is implemented in digital FPGA/DSP platform [5], [13], thus, the whole transmitter is reconfigurable.

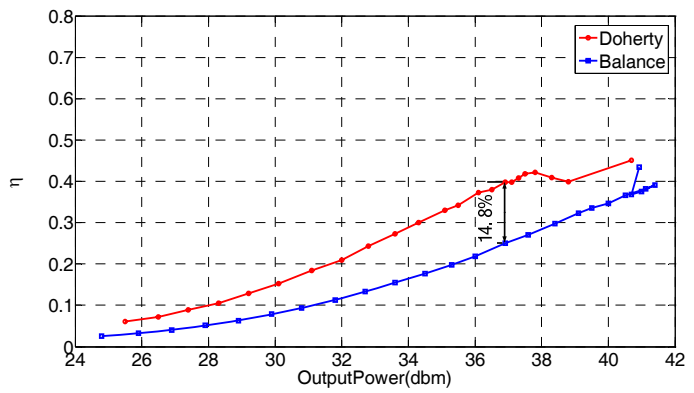

(a)

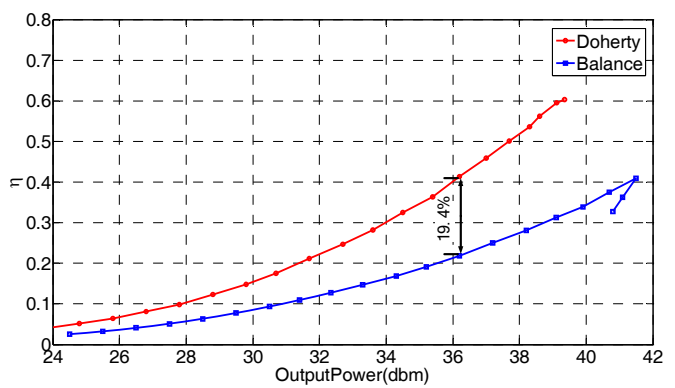

(b)

Fig. 4 Drain efficiency at: (a) first frequency of operation, (b) second frequency of operation.

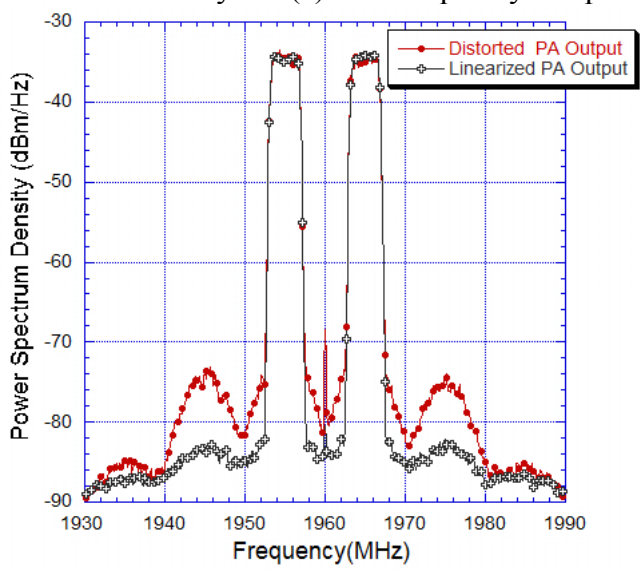

(a)

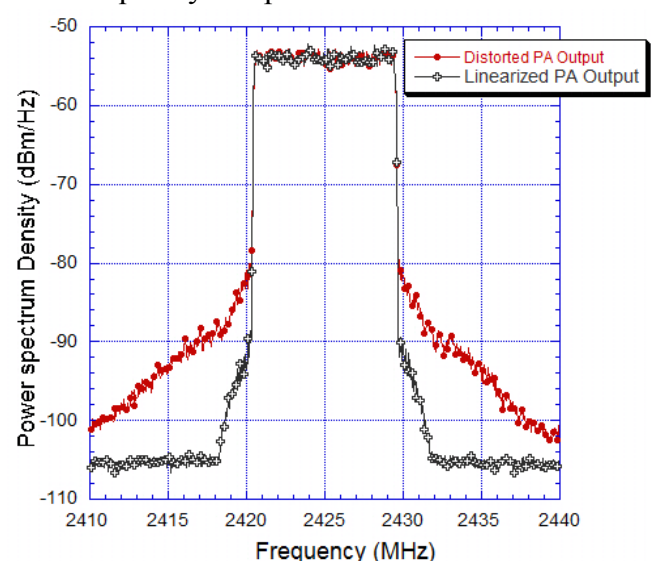

(b)

Fig. 5 Performance in terms of linearization: (a) 2 Carrier 101 WCDMA, (b) $10 \mathrm{MHz}$ WiMax signal.

\section{Conclusion}

For the sake of reconfigurability in the SDR based transmitter, in order to maintain the performance in terms of linearity and efficiency, a FPGA/DSP based implementation of digital predistortion and a multi-band Doherty PA can be considered as optimum configuration. The current state of art for the multi-band Doherty design still needs further research in terms of certain components such as multi-band delay lines and robust multi-band matching techniques. The design also needs to be optimized for concurrent-operation, which finds application in smooth transition of current 
existing communication standards to $4 \mathrm{G}$ standards and hence needing backward compatibility. For such purpose, a concurrent digital predistortion also presents a promising research field.

\section{References}

1. P. Kenington, RF and Baseband Techniques for Software Defined Radio. Boston: Artech House, 2005, ch. 5, pp. 183-229.

2. F.M. Ghannouchi, "Power Amplifier and Transmitter Architectures for Software Defined Radio Systems," Circuit \& system magazine IEEE, vol. 10, no. 4, Dec 2010, pp. 56-63.

3. F. H. Raab, "Efficiency of Doherty RF Power Amplifier System," Trans. Broadcasting IEEE, vol. BC-33, no. 3, Sep. 1983, pp. 77-83.

4. B. Kim, J. Kim, I. Kim, J. Cha, “The Doherty Amplifier,” Microwave Magazine IEEE, vol. 7, no. 5, Oct. 2006, pp. 42-50.

5. E. Jeckeln, F. M. Ghannouchi and M. Sawan, “A new adaptive predistortion technique using software-defined radio and DSP technologies suitable for base station 3G power amplifiers", Trans. Microw. Theory Tech. IEEE, vol. 52, no. 9, Sep. 2004, pp. 2139-2147.

6. K. Rawat, M. Rawat, F. M. Ghannouchi, “Compensating I-Q Imperfections in Hybrid RF/Digital Predistortion With an Adapted Lookup Table Implemented in an FPGA”, Trans. Circuit \& System-II. IEEE, vol. 57, no. 5, May. 2010, pp. 389-393.

7. K.K.M. Cheng and F.L. Wong, "A novel approach to the design and implementation of dual-band compact planar $90^{\circ}$ branch-line coupler," Trans. Microw. Theory Tech. IEEE, vol. 52, no. 11, Nov. 2004, pp. 2458-2463.

8. K. Rawat, F.M. Ghannouchi, M. Rawat, M.S. Hashmi, “Analysis of Frequency Selective Impedance Loading of Transmission Lines for Dual Band Couplers," in International Journal of RF MiCAE, Wiley (under publication).

9. C. Monzon, “A small dual-frequency transformer in two sections," Trans. Microw. Theory Tech. IEEE, vol. 51, Apr. 2003, pp.1157-1161.

10. P. Colantonio, F. Feudo, F. Giannini, R. Giofre, Rocco, L. Piazzon, "Design of a dual-band GaN Doherty amplifier," 18th International Conference on Microwave Radar and Wireless Communications (MIKON), 2010, pp. 14.

11. Xi Li, W. Chen, Z. Zhang, Z. Feng, X. Tang, K. Mouthaan, “A Concurrent Dual-Band Doherty Power Amplifier," Proc. of Asia Pacific Microwave Conf. 2010, pp. 1-4.

12. M. Rawat, K. Rawat and F.M. Ghannouchi, "Adaptive Digital Predistortion of Wireless Power Amplifiers/Transmitters Using Dynamic Real-Valued Focused Time Delay Line Neural Networks", Trans. Microw. Theory Tech. IEEE, vol. 58, no. 1, Jan. 2010, pp. 95-104.

13. A. Kwan, M. Helaoui, S. Boumaiza, M. Smith and F. M. Ghannouchi, "Wireless Communications Transmitter Performance Enhancement Using Advanced Signal Processing Algorithms Running in a Hybrid Dsp/Fpga Platform," The Journal of VLSI Signal Processing Systems for Signal, Image, and Video Technology, vol. 56, no. 1, Feb 2009, pp. 187-198. 